Aim of the study: To investigate the efficacy of evaluating prognosis and response to lung cancer treatment using M30 and M65 antigens, which are indicators of necrosis.

Material and methods: Forty-eight patients with lung cancer, who were planned to receive neoadjuvant chemotherapy, and 38 healthy volunteers were enrolled in the study. Using M30 and M65 levels, cytokeratin 18 levels were measured twice: before and 48 hours after the first chemotherapy treatment. Apoptotic and total necrosis levels were determined by measuring the $\mathrm{M} 65$ and $\mathrm{M} 30$ levels.

Results: The M30 and M65 antigen levels in the patient group were significantly higher than in the control group $(p<0.001)$. The M30 and M65 antigen levels were significantly higher 48 hours after the chemotherapy compared with before the chemotherapy $(p<0.001)$. There were no significant differences in $\mathrm{M} 65$ levels between patients who responded to treatment and patients who progressed. The M30 levels increased significantly in patients with disease progression ( $p=$ 0.694 and $p=0.024$, respectively). No significant differences in serum M30 and M65 antigen levels were found when compared between the surviving and deceased patients ( $p=0.126$ and $p=0.340$, respectively).

Conclusions: A significant increase was detected in serum M30 and M65 levels in patients with lung cancer. There was a greater increase in serum M30 levels in patients who did not respond to the chemotherapy. This result gives rise to the thought that evaluating apoptosis and total necrosis through M30 and M65 measurements alone only in patients receiving neoadjuvant chemotherapy would be insufficient for specifying the effectiveness of the treatment.

Key words: lung cancer, neoadjuvant treatment, prognosis, cytokeratin.

Contemp Oncol (Pozn) 2019; 23 (4): 208-213 DOI: https://doi.org/10.5114/wo.2019.91539

\section{The roles of M30 and M65 in the assessment of treatment response and prognosis in patients with non-small cell lung cancer, who receive neoadjuvant treatment}

\author{
Belkıs Nihan Coskun ${ }^{1}$, Oguzhan Sıtkı Dizdar², Seniz Korkmaz ${ }^{3}$, \\ Engin Ulukaya ${ }^{3}$, Turkkan Evrensel ${ }^{4}$
}

${ }^{1}$ Department of Internal Medicine, Uludağ University Medical School, Bursa, Turkey 2Department of Internal Medicine, Kayseri Training and Research Hospital, Kayseri, Turkey

${ }^{3}$ Department of Clinical Biochemistry, Uludağ University Medical School, Bursa, Turkey ${ }^{4}$ Department of Medical Oncology, Uludağ University Medical School, Bursa, Turkey

\section{Introduction}

One of the most important aims in the administration of medication in lung cancer, which causes mortality related to cancer, is to expedite apoptosis in neoplastic cells [1]. Thus, monitoring necrosis may be used to determine the efficacy of anti-cancer treatment. However, to our knowledge, no indicators have been proven to be reliable in this regard in the relevant literature.

Cytokeratin 18 (CK-18) is the primary element of epithelial and endothelial filaments. It is produced by proliferating cells and is excreted into circulation when the membrane disintegrates during necrosis. Caspase causes CK-18 to fracture only within cells that die due to apoptosis [2]. The M30 monoclonal antibody recognises the fractured part (M30 antigen), particularly during apoptosis of CK-18; therefore, CKs could be used as an apoptotic indicator [3]. The M65 antigen levels are related with total CK-18, which accounts for total necrosis.

In monitoring necrosis, measuring the amount of substances produced and excreted in the blood during necrosis is a simple method. Cytokeratins, which are one of the indicators measured in blood, have been suggested to have advantages owing to their ability to show a distinction between apoptosis and necrosis [4]. Normally, CKs in healthy individuals have lower circulating levels in the blood. A significant increase is observed in cancers related to epithelial cells [5], which eliminates the likelihood of serum levels being under the effects of non-epithelial organs such as bone marrow, given that bone marrow is the most affected organ during chemotherapy, thereby suggesting the significance and advantage of CK-18 measurements when monitoring the response of cells during cancer treatment [6].

This aim of the present study was to investigate the efficacy of M30 and M65 antigen measurements in the evaluation of prognosis and response to treatment in lung cancer. Our study is the first to examine the effectiveness of treatment among patients receiving neoadjuvant chemotherapy, who had not yet undergone surgery, and the association between apoptosis and necrosis parameters.

\section{Material and methods}

Patients aged over 18 years, who were diagnosed as having lung cancer and were planned to receive neoadjuvant chemotherapy treatment were 
enrolled in the study group. Patients with secondary malignancies, patients with renal and hepatic disorders, and those with poor bone marrow function were excluded. The control group comprised volunteer individuals who were aged over 18 years, were not under any medication, and had no common diseases. Ethics committee approval was obtained from the Board of Research and Ethics of the Medical Faculty. Written, informed consent was obtained from all participants.

For measuring serum CK-18 levels, samples were taken from the patient group twice: first, before the first chemotherapy treatment; and second, 48 hours after the chemotherapy. Blood was only drawn once from the control group. The second blood sample scheduled for 48 hours after treatment could not be taken from five patients in the study group, one patient was referred to another institution due to cranial infarction after chemotherapy, and four patients were out of town.

The TNM staging system was used to stage the patients according to the American Joint Committee on Cancer, and computed tomography (CT) monitoring was performed primarily. However, in conditions where CT was insufficient in staging, positron emission tomography (PET/CT) was used.

When the treatment finished, the response of the patients to chemotherapy was defined according to the Response Evaluation Criteria in Solid Tumours (RECIST) version 1.1. According to this, complete reduction of the tumour is considered a full response, a 30\% decrease in tumour size is considered partial response, a $20 \%$ increase in tumour size is regarded as progressive disease, and small changes outside these criteria are evaluated as stable disease. The ages, sexes, histologies, TNM stages, chemotherapy protocols, adverse effects due to chemotherapy, and best response parameters of our patients (partial response, stable disease, progressive disease) were evaluated.

The serum CK18 levels were measured using an M65 enzyme-linked immunosorbent assay (ELISA) (Peviva, AB-Sweden) and M30-ELISA (Peviva, AB-Sweden) kits. The M30 ELISA is a quantitative specific and sensitive measurement method for caspase fractional CK-18
(CK-18Asp396-NE: M30 neo-epitope). The M65-ELISA kit was used to measure total CK-18. The apoptotic and total necrosis levels could be identified using the M65 and M30 ELISA kits.

With the effects of caspase, which is an enzyme group activated only in apoptotic cells, CK-18 forms fractured CK-18 (CK-18-Asp396) [7]. The M30 monoclonal antibody provides use for CKs as apoptotic indicators, particularly by recognising the fractured fragment (M30 antigen) in Asp396 of CK-18 (Fig. 1). Thus, the fractured CK-18, which is an indicator specific to apoptosis, is revealed by using the ELISA method. The presence of M30 antibodies recognising these specific CK-18 fragments indicates the presence of apoptosis.

\section{Statistical analysis}

The normality and homogeneity of the data were evaluated using the Kolmogorov-Smirnov test and Levene's test, respectively. Comparisons between groups for continuous variables were performed using Student's t-test (normal distribution) or the Mann-Whitney $U$ test (non-normal distribution). Fisher's test or the $\chi^{2}$ test was used for all categorical data. A paired sample $t$ test or the Wilcoxon test was conducted to compare the measurements of patients before and after chemotherapy. The associations between the recovery period and M30 and M65 ratios before the treatment were investigated using Pearson's correlation analyses. Basal serum M30 and M65 antigen levels according to stages and clinical response to chemotherapy were compared using the Kruskal-Wallis test. All calculations were conducted using the Statistical Package for the Social Sciences version 16.0 statistical package (SPSS, Chicago, IL, USA). The $p<0.05$ was considered statistically significant.

\section{Results}

Forty-eight patients (women $n=2,4.2 \%$; men $n=46$, 95.8\%) who were diagnosed as having lung cancer and were planned to receive neoadjuvant chemotherapy and 38 healthy volunteers (women $n=2,5.3 \%$; men $n=36$,

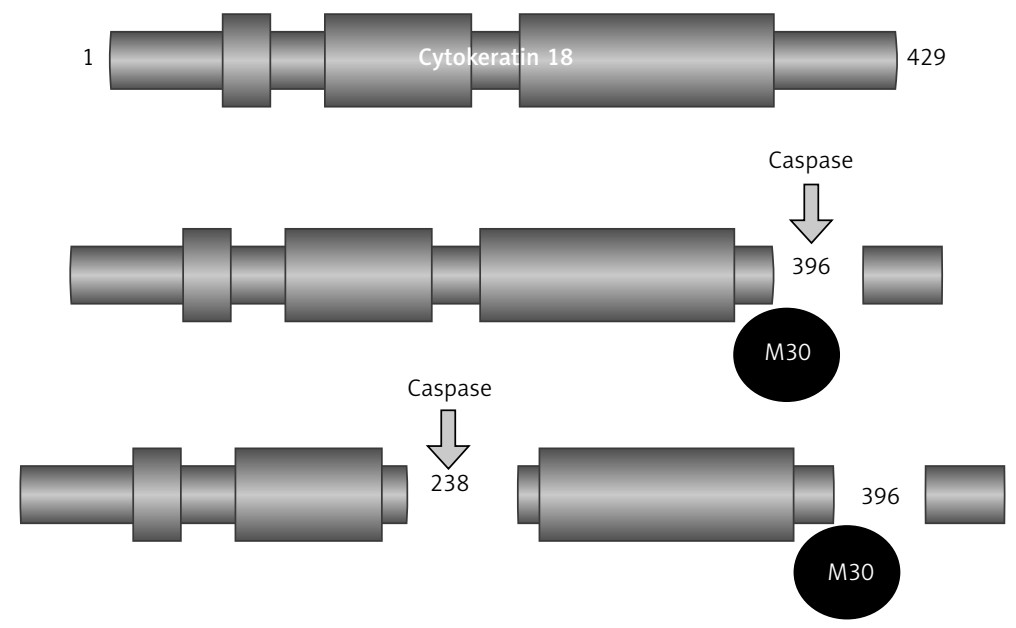

Fig. 1. Breakdown of cytokeratin 18 with caspases and M30 antibody recognises these regions 
94.7\%) participated in this study. The average age of the patients was $57.5 \pm 9.3$ years. The majority (87.5\%) of the patients had histologic type squamous cell carcinoma, 8.3\% had adenocarcinoma, and $4.2 \%$ had large cell carcinoma. In terms of tumour stage, $47.9 \%$ had stage IIIA cancer, $39.6 \%$ had stage IIIB cancer, and $2.5 \%$ had stage IV disease. Thirty-six (75\%) patients received docetaxel + cisplatin, nine $(18.8 \%)$ received paclitaxel + carboplatin treatment, and three $(6.3 \%)$ received gemcitabine + cisplatin treatment. According to the chemotherapy protocols, when the M30 and M65 levels were compared between the 36 patients receiving docetaxel + cisplatin treatment and the nine patients receiving paclitaxel + carboplatin treatment before (baseline) the treatment, there was no significant difference ( $p=0.267$ and $p=0.686$, respectively).

The serum M30 and M65 antigen levels of the control group and patient group before and 48 hours after the chemotherapy treatment are presented in Table 1 . The M30 and M65 antigen levels of the patient group were significantly higher than in the control group. The M30 and M65 antigen levels were significantly higher 48 hours after the chemotherapy than before the chemotherapy.

According to the RECIST criteria, the response rate to the neoadjuvant treatment was 60.5\%; four patients (9.3\%) had a full response, and $22(51.2 \%)$ had a partial response. After the treatment, stable disease was detected in three patients (7\%) and progression was observed in 14 patients (32.5\%). No significant differences were found when the M30 and M65 levels before treatment were compared among patients who responded to treatment and those with disease progression ( $p=0.795, p=0.097$, respectively) (Table 2). There were no differences in baseline M30 and M65 antigen levels according to the patients' stages (Table 2).

The average survival time was $14.75 \pm 9.51$ months and the 12 -month survival probability was $68.4 \%$. According to Pearson's correlation analysis performed between the survival time and M30 and M65 levels before treatment, no significant relation was revealed $(p=0.678$ and $p=0.055$, respectively).
The levels of serum M30 and M65 in patients after chemotherapy were calculated to indicate the percentage (\%) change from before the chemotherapy treatment. The percentage change was calculated using the following formula:

variation $\%=100 \times($ M30 or M65 levels two days after chemotherapy - M30 or M65 levels before chemotherapy) / M30 or M65 levels before chemotherapy

The findings showed that the M30 levels in patients who responded to the treatment increased by $34 \%$, and the M65 levels increased by $68 \%$. The M30 levels in patients with disease progression increased by $78 \%$, and the $M 65$ levels increased by $54 \%$. When we take the percentage variation of the patients into account, the findings indicated that there was no difference in M65 levels when compared between patients who and did and did not respond to treatment. By contrast, the M30 levels increased significantly in patients with disease progression ( $p=0.694$ and $p=0.024$, respectively).

Eighteen out of the 48 patients who were treated with neoadjuvant chemotherapy underwent surgery after the treatment. A full pathologic response was found in eight of the 18 patients who underwent surgery. The average serum M30 antigen level in patients with a full response was $142.75 \pm 58.97$, and the average serum M65 antigen level was $169.75 \pm 85.63$. The average M30 antigen level in 10 patients who did not respond pathologically was $140.90 \pm 54.16$, and the average serum M65 antigen level was $158.20 \pm 52.24$. When the baseline M30 and M65 ratios of patients who did and did not respond totally were compared, no significant variation was revealed ( $p=0.965$ and $p=0.203$, respectively) (Table 3 ). No significant variation was revealed when the baseline serum M30 and M65 antigen levels of patients who did and did not have a full pathologic response were compared ( $p=0.696$ and $p=$ 0.274 , respectively).

Twenty-three of the 48 patients in the study group died. The average baseline serum M30 level was $171.64 \pm 139.71$ in the surviving 25 patients, whereas it was $155.34 \pm 71.3$

Table 1. Comparison of serum M30 and M65 levels of patient and control groups

$\begin{array}{lcccc}\text { Variables } & \begin{array}{c}\text { Control group } \\ (n=38)\end{array} & \begin{array}{c}\text { Before chemotherapy (baseline) } \\ (n=48)\end{array} & \begin{array}{c}\text { After chemotherapy (48 } \\ (n=43)\end{array} & p \\ \text { M30 level } & 76(65-127) & 131(109-186) & 176(136-249) & <0.001^{*},<0.001^{* *} \\ \text { M60 level } & 285(226-416) & 397(278-509) & 575(416-778) & <0.05^{*},<0.001^{* *}\end{array}$

Data are expressed as median (including the lower and upper quartiles), Mann-Whitney U test was used for comparisons between the groups for M30 and M60 level, and Wilcoxon test was conducted to compare the measurements of patients before and after chemotherapy, ${ }^{*}$ comparison of serum M30 or M60 antigen levels of healthy control group and patients who had not yet received chemotherapy, " comparison of serum M30 or M60 antigen levels before and after chemotherapy.

Table 2. Baseline serum M30 and M65 antigen levels according to stages and clinical response to chemotherapy

\begin{tabular}{|c|c|c|c|c|c|c|c|}
\hline Variables & $\begin{array}{l}\text { Patients responding } \\
\text { to treatment } \\
(n=29)\end{array}$ & $\begin{array}{l}\text { Patients with } \\
\text { progression } \\
(n=14)\end{array}$ & $p$ & Stage $3 A$ & Stage 3B & Stage 4 & $p$ \\
\hline Serum M30 level & 138 (111-195) & $126(119-175)$ & 0.795 & 127 (113-194) & $132(114-156)$ & $180.0 \pm 57.1$ & 0.435 \\
\hline Serum M65 level & $371(258-507)$ & $501(316-733)$ & 0.097 & $353(226-497)$ & $373(276-554)$ & $543.0 \pm 82.3$ & 0.059 \\
\hline
\end{tabular}

Data are expressed as the mean \pm SD or median (including the lower and upper quartiles), Mann-Whitney $U$ test was used for comparisons between the groups for M30 and M60 level and basal serum M30 and M65 antigen levels according to stages and clinical response to chemotherapy were compared using the Kruskal-Wallis test 
Table 3. Baseline serum M30 and M65 antigen levels in patients according to pathologic response and survival

\begin{tabular}{ccccccc|} 
Variables & $\begin{array}{c}\text { Pathologically responsive } \\
\text { patients } \\
(n=8)\end{array}$ & $\begin{array}{c}\text { Patients with no pathologic } \\
\text { complete response } \\
(n=10)\end{array}$ & $p$ & $\begin{array}{c}\text { Surviving patients } \\
(n=25)\end{array}$ & $\begin{array}{c}\text { Deceased } \\
\text { patients } \\
(n=23)\end{array}$ & $p$ \\
M30 level & $142.75 \pm 58.97$ & $140.90 \pm 54.16$ & 0.965 & $171.64 \pm 139.71$ & $155.34 \pm 71.30$ & 0.959 \\
M65 level & $169.75 \pm 85.63$ & $158.20 \pm 52.24$ & 0.203 & $419.68 \pm 253.86$ & $455.56 \pm 68.15$ & 0.584 \\
\hline
\end{tabular}

in the 23 patients who died. The average M65 level in the surviving patients was $419.68 \pm 253.86$, and it was 455.56 \pm 268.154 in those who died. However, when the baseline ratios of M30 and M65 of the surviving and deceased patients were compared, there was no significant variations ( $p=0.959$ and $p=0.584$, respectively) (Table 3). A 33\% increase in serum M30 antigen levels was detected in the surviving 24 patients after treatment, whereas a 63\% increase was present after treatment in the 19 patients who died. A 72\% increase in serum M65 levels was found after treatment in the 24 surviving patients, whereas the in crease was 55\% in the 19 patients who died. However, no significant differences in serum M30 and M65 antigen levels were found when compared between the survivors and patients who died ( $p=0.126$ and $p=0.340$, respectively).

\section{Discussion}

Today, detecting whether cancer treatment is effective in the early period is important in order to be able to make earlier modifications to the treatment protocol. Cancer treatment causes tumour necrosis through apoptosis [8]; therefore, apoptosis is an important parameter in evaluating the effectiveness of cancer treatment. Measuring macromolecules in the serum released from the necrosis of tumours during chemotherapy is accepted as an effective and inexpensive method of monitoring the efficacy of treatment. Fractured CK-18 (M30) is only formed as a result of fracture by caspases, which are protease enzymes specific to the total CK-18 apoptosis in necrosis. It is considered that the M30 fractions of cytokeratins indicating apoptotic necrosis and the M65 fraction indicating total necrosis can be used to evaluate cancer necrosis [2]. In our study, using ELISA, we measured M30, which is one of the products generated from apoptotic cells, and M65 antigen levels indicating total necrosis, and we aimed to predict the response to neoadjuvant treatment among patients with lung cancer. We also sought to evaluate the use of these measurements as prognostic and predictive parameters. We should highlight that several studies have measured M30 and M65 antigen levels in patients with lung cancer; however, incompatible results have been reported. This situation may have resulted from tumours that have completely different features and the medicines used, which have different tumour-killing mechanisms. In our study, the clinical monitoring parameters of M30 and M65 levels were evaluated for the first time in patients receiving only neoadjuvant treatment and their relationship with chemotherapy, and an objective evaluation was performed according to whether a pathologic response was present after the patients underwent surgery. Thus, we tried to objectively clarify this controversial subject.

In the study by Kramer et al. [2], an increase was found in the number of apoptotic cells 24 and 48 hours after breast cancer treated with cisplatin. The total CK-18 and fractured CK-18 levels in local bloods of patients with malignant endometrial cancer were detected higher than in peripheral blood levels. These results were interpreted as meaning that total CK-18 and fractured CK-18 were generated by tumours. In studies conducted on patients with lung cancer, head and neck tumours, and breast cancer, the findings showed that patients had higher M30 and M65 antigen levels than the controls [3, 9, 10]. In our study, we detected higher M30 and M65 levels in patients with lung cancer than in the control group, which supports our hypothesis.

Given that there is not always a dominant necrosis type during cancer treatment, both apoptosis and total necrosis must be monitored. Therefore, we evaluated M30 and total necrosis in patients with lung cancer by measuring M65, and we revealed the relationship between necrosis and other clinical parameters.

Öztürk et al. considered that serum M30 levels in head and neck tumours might have prognostic importance [9]. Ueno et al. detected that the M30 antigen levels of patients with breast cancer, who were in poor clinical condition, were higher than in healthy patients [3]. However, no relation among the prognoses of the patients and the $\mathrm{M} 30$ antigen levels was revealed. In a study on patients with gastric cancer, M30 and M65 antigen levels were related to non-progressive recovery [11]. In another study conducted among patients with advanced gastric cancer, it was specified that M30 levels were beneficial in estimating recovery and the treatment response of the tumour [12]. M30 antigen levels are considered as an interesting marker in terms of residual tumour after surgery in colorectal cancers and early recurrence. It has also been suggested in various studies that M65 antigen levels might indicate the common disease $[13,14]$. In our research, no relationship between $\mathrm{M} 30$ and M65 antigen levels or between recovery and disease stage were detected. However, as the stage of the disease increased, albeit not statistically significantly, we detected an increase in serum M65 levels. For detecting relations between long-term prognosis and cytokeratins, long-term, monitored, prospective studies are required.

In the study by Ulukaya et al., the 24- and 48-hour serum $\mathrm{M} 30$ levels were high in patients who received cisplatin-based chemotherapy [10]. No relationship between M30 antigen levels and the recovery period was detected after chemotherapy. However, serum M65 levels were not 
evaluated in their study. Hou et al. considered that serum M30 and M65 antigen levels were important prognostically in terms of recovery in patients with small-cell lung cancer [15]. Within the same study, the findings showed that serum M30 and M65 antigen levels peaked at the $48^{\text {th }}$ hour and decreased after the $22^{\text {nd }}$ day, which indicated the response of the tumour. The increase at the $48^{\text {th }}$ hour was associated with early response and toxicity. In our study, after similar chemotherapy, the M30 and M65 antigen levels measured at the $48^{\text {th }}$ hour had increased from baseline. However, we considered that the baseline M30 and M65 levels were not significant in terms of recovery.

In our study, the M30 and M65 levels in patients who did and did not respond to treatment increased after chemotherapy. The increase in M65 levels of all patients who did and did not respond treatment was twice as high as the total M30 level (68\% and 34\%, respectively, before treatment). The increase in M65 levels of patients who failed to respond to treatment was lower than the total M30 level (54\% and 78\%, respectively, of the value before treatment). This finding suggests that the type of necrosis, rather than apoptosis after chemotherapy, in patients with lung cancer is more important.

Chu et al. estimated that apoptotic necrosis was less in patients with small sub-celled lung cancer receiving paclitaxel chemotherapy, but necrotic necrosis came into prominence; these results are compatible with those of our study [16]. However, when the M30 and M65 levels of patients who did and did not respond to treatment were compared, although a significant difference was not detected, the findings showed that M30 ratios significantly increased more in patients who failed to respond to treatment. Demiray et al. detected a significant increase in the $24^{\text {th }}$ and $48^{\text {th }}$ hour serum M30 levels of patients with local advanced breast cancer, who received neoadjuvant treatment and responded; however, they stated that the levels did not change in patients who failed to respond [17].

It has been considered that the induction of apoptosis after treatment enables patients with cancer to be treated much better. In line with this opinion, it has been reported that there was a positive relation between the clinical response of patients with local advanced breast cancer who received neoadjuvant chemotherapy and increased apoptosis [18]. However, our findings are incompatible with this. Our findings showed that the increase in M30 of patients who failed to respond to treatment might correlate with the treatment response of the tumour. The results of a study conducted on patients with testicular germ cell cancer also support our notion. A correlation between post-treatment serum M30 and M65 levels and prognostic indicators were detected. This was considered probably to be an indication of the treatment response of the tumour. In the end, as our study indicates, the findings showed that serum M30 and M65 levels significantly increased after treatment in patients with poor prognosis [19]. Ustaalioglu et al. indicated that M30 and M65 levels were incapable of estimating response after chemotherapy in patients with non-small cell lung cancer [20]. However, in their study, they found that high levels of M30 were an independent risk factor in terms of recovery. These re- sults give rise to the thought that M30 and M65 levels do not provide sufficient data to evaluate treatment response in lung cancer. On the other hand, Badzio et al. found that high expression of keratin-specific antibodies AE1/AE3 is a favourable prognostic factor in surgically treated small cell lung cancer [21]. Due to our data being the first in patients with lung cancer, who received neoadjuvant treatment, there is a need for further studies in which tumours with different treatment responses are considered, and in creating different responses to chemotherapy [22].

One of the most important limitations in our study is the small cohort of patients. The other limitation is the non-measurement of M30 and M65 levels after the first chemotherapy, and the levels before and after other chemotherapies. Accordingly, we could not monitor the changes in time of M30 and M65 levels.

\section{Conclusions}

In our study, a significant increase was detected in serum M30 and M65 levels in patients with lung cancer when compared with healthy individuals, suggesting that M30 and M65 could be used as predictive markers. M30 and M65 must be measured together in patients with lung cancer in the evaluation of necrosis, given that our findings indicate that chemotherapy and non-apoptotic necrosis are more prominent. Serum M30 levels increased much more in patients who failed to respond to chemotherapy, when compared with patients who responded. This result suggests that evaluating apoptosis and total necrosis through M30 and M65 measurements alone in patients receiving neoadjuvant chemotherapy will be insufficient to specify the effectiveness of treatment. No relation was detected between the recovery of M30 and M65 levels and various prognostic indicators. These findings suggest that many factors, particularly tumour treatment response apart from apoptosis, influence the effectiveness of treatment and prognosis.

The authors declare no conflict of interest.

\section{References}

1. Fisher DE. Apoptosis in cancer therapy: Crossing the threshold. Cell 1994; 78: 539-542.

2. Kramer G, Erdal H, Mertens HJ, et al. Differentiation between cell death modes using measurements of different soluble forms of extracellular cytokeratin 18. Cancer Res 2004;64:1751-56.

3. Ueno T, Toi M, Biven K, Bando H, Ogawa T, Linder S. Measurement of an apoptotic product in the sera of breast cancer patient. Eur J Cancer 2003; 39: 769-774.

4. Caulin C, Salvesen GS, Oshima RG. Caspase cleavage of keratin 18 and reorganization of intermediate filaments during epithelial cell apoptosis. J Cell Biol 1997; 138: 1379-1394.

5. Moll R, Franke WW, Schiller DL, Geiger B, Krepler R. The catalog of human cytokeratins: patterns of expression in normal epithelia, tumors and cultured cells. Cell 1982; 31: 11-24.

6. Stasiak PC, Purkis PE, Leigh IM, Lane EB. Keratin 19: Predicted amino acid sequence and broad tissue distribution suggest it evolved from keratinocyte keratins. J Invest Dermatol 1989; 92: 707-716.

7. Leers MP, Kolgen W, Bjorklund V, et al. Immunocytochemical detection and mapping of a cytokeratin 18 neo-epitope exposed during early apoptosis. J Pathol 1999; 187: 567-572. 
8. Hickman JA, Beere HM, Wood AC, Waters CM. Mechanisms of cytotoxicity caused by antitumour drugs. Toxicol Lett 1992; 64-65: 553-561.

9. Öztürk B, Coskun U, Sancak B, Yaman E, Buyukberber S, Benekli M. Elevated serum levels of M30 and M65 in patients with locally advanced head and neck tumors. Int Immunopharmacol 2009; 9: 645-648.

10. Ulukaya E, Yilmaztepe A, Akgoz S, Linder S, Karadag M. The levels of caspase-cleaved cytokeratin 18 are elevated in serum from patients with lung cancer and helpful to predict the survival. Lung Cancer 2007; 56: 399-404.

11. Bilici A, Ustaalioglu BB, Ercan S, Orcun A, Seker M, Salepci T, Gumus M. Is there any impact of plasma M30 and M65 levels on progression-free survival of patients with advanced gastric cancer? Cancer Chemother Pharmacol 2011; 68: 309-316.

12. Yaman E, Coskun U, Sancak B, Buyukberber S, Ozturk B, Benekli M. Serum M30 levels are associated with survival in advanced gastric carcinoma patients. Int Immunopharmacol 2010; 10: 719-722.

13. Ausch C, Buxhofer-Ausch V, Olszewski U, Hinterberger W, Ogris E, Schiessel R, Hamilton G. Caspase-cleaved cytokeratin 18 fragment (M30) as marker of postoperative residual tumor load in colon cancer patients. Eur J Surg Oncol 2009; 35: 1164-1168.

14. Ausch C, Buxhofer-Ausch V, Olszewski U, Schiessel R, Ogris E, Hinterberger W, Hamilton G. Circulating cytokeratin 18 fragment M65 - A potential marker of malignancy in colorectal cancer patients. J Gastrointest Surg 2009; 13: 2020-2026.

15. Hou JM, Greystoke A, Lancashire L, et al. Evaluation of circulating tumor cells and serological cell death biomarkers in small cell lung cancer patients undergoing chemotherapy. Am J Pathol 2009; 175: 808-816.

16. Chu T, Jiang L, Ying W, Han B. M30/M65 ratio predicts the outcome of paclitaxel chemotherapy for NSCLC. Clin Transl Oncol 2017; 19: 326-331.

17. Demiray M, Ulukaya E, Arslan M, et al. Response to neoadjuvant chemotherapy in breast cancer could be predictable by measuring a novel serum apoptosis product, caspase-cleaved cytokeratin 18: a prospective pilot study. Cancer Invest 2006; 24: 669-676.

18. Tiezzi DG, De Andrade JM, Cândido dos Reis FJ, et al. Apoptosis induced by neoadjuvant chemotherapy in breast cancer. Pathology 2006; 38: 21-27.

19. de Haas EC, di Pietro A, Simpson KL, et al. Clinical evaluation of M30 and M65 ELISA cell death assays as circulating biomarkers in a drug-sensitive tumor, testicular cancer. Neoplasia 2008; 10: 1041-1048.

20. Ustaalioglu BB, Bilici A, Ercan S, Seker M, Orcun A, Gumus M. The prognostic importance of changing serum M30 and M65 values after chemotherapy in patients with advanced-stage non-smallcell lung cancer. Med Oncol 2013; 30: 551.

21. Badzio A, Czapiewski P, Gorczyński A, Szczepańska-Michalska K, Haybaeck J, Biernat W, Jassem J. Prognostic value of broad-spectrum keratin clones AE1/AE3 and CAM5.2 in small cell lung cancer patients undergoing pulmonary resection. Acta Biochim Pol 2019, 66: 111-114.

22. Jelonek K, Widłak P. Metabolome-based biomarkers: their potential role in the early detection of lung cancer. Contemp Oncol (Pozn) 2018; 22: 135-140.

\section{Address for correspondence}

\section{Oguzhan Sıtkı Dizda}

Department of Internal Medicine

Kayseri Training and Research Hospital

Atatürk Ave. Hastane St. No. 78

38010 Kayseri, Turkey

e-mail: osdizdar@gmail.com

Submitted: 14.10 .2019

Accepted: 27.11.2019 